Damping of vibrational excitations in glasses at terahertz frequency: The case of 3methylpentane

Giacomo Baldi, Paola Benassi, Aldo Fontana, Andrea Giugni, Giulio Monaco, Michele Nardone, and Flavio Rossi

Citation: The Journal of Chemical Physics 147, 164501 (2017); doi: 10.1063/1.4998696

View online: $\mathrm{http}: / / d x . d o i . o r g / 10.1063 / 1.4998696$

View Table of Contents: http://aip.scitation.org/toc/jcp/147/16

Published by the American Institute of Physics

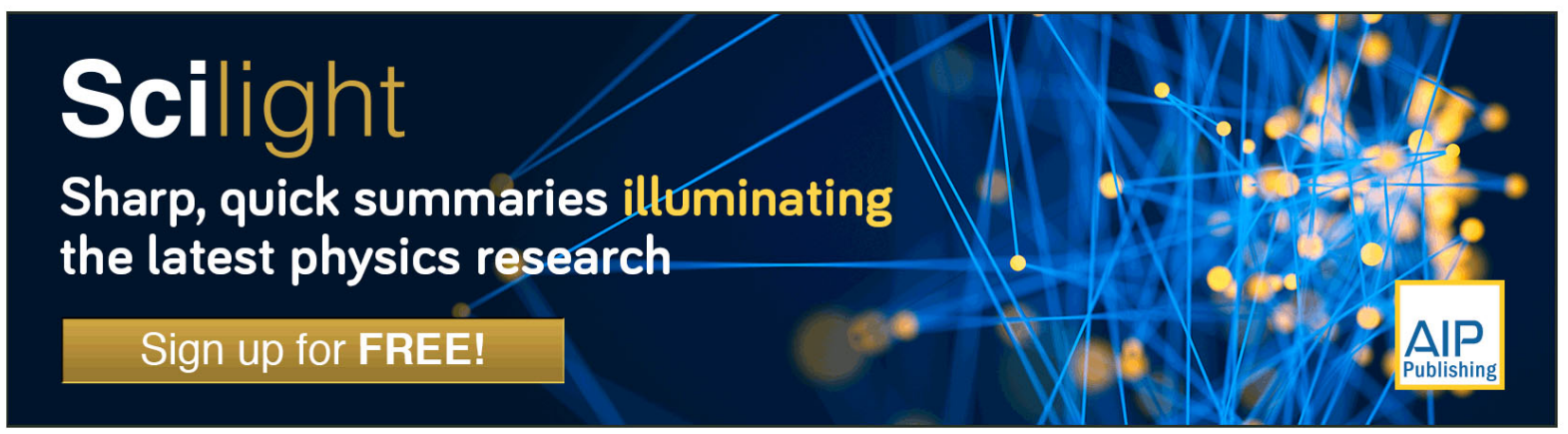




\title{
Damping of vibrational excitations in glasses at terahertz frequency: The case of 3-methylpentane
}

\author{
Giacomo Baldi, ${ }^{1, a)}$ Paola Benassi, ${ }^{2}$ Aldo Fontana, ${ }^{1}$ Andrea Giugni, ${ }^{3}$ Giulio Monaco, ${ }^{1}$ \\ Michele Nardone, ${ }^{2}$ and Flavio Rossi ${ }^{1}$ \\ ${ }^{1}$ Dipartimento di Fisica, Università di Trento, 38050 Povo, Trento, Italy \\ ${ }^{2}$ Dipartimento di Scienze Fisiche e Chimiche, Università degli Studi dell'Aquila, 67100 L'Aquila, Italy \\ ${ }^{3}$ PSE and BESE Divisions, King Abdullah University of Science and Technology, \\ Thuwal 23955-6900, Saudi Arabia
}

(Received 2 August 2017; accepted 10 October 2017; published online 24 October 2017)

\begin{abstract}
We report a compared analysis of inelastic X ray scattering (IXS) and of low frequency Raman data of glassy 3-methylpentane. The IXS spectra have been analysed allowing for the existence of two distinct excitations at each scattering wavevector obtaining a consistent interpretation of the spectra. In particular, this procedure allows us to interpret the linewidth of the modes in terms of a simple model which relates them to the width of the first sharp diffraction peak in the static structure factor. In this model, the width of the modes arises from the blurring of the dispersion curves which increases approaching the boundary of the first pseudo-Brillouin zone. The position of the boson peak contribution to the density of vibrational states derived from the Raman scattering measurements is in agreement with the interpretation of the two excitations in terms of a longitudinal mode and a transverse mode, the latter being a result of the mixed character of the transverse modes away from the center of the pseudo-Brillouin zone. Published by AIP Publishing. https://doi.org/10.1063/1.4998696
\end{abstract}

\section{INTRODUCTION}

The lack of translational periodicity characteristic of disordered solids such as glasses and the related difficulties encountered in describing their microscopic dynamics with respect to crystals pose several intriguing issues. For this reason, in the last years, a strong experimental ${ }^{1-26}$ and theoret$\mathrm{ical}^{27-40}$ effort has been made. In this context, inelastic X-ray and neutron scattering investigations $\mathbf{s}^{1,9-13,15,21}$ have obtained a very important and stimulating evidence, namely, the existence, also in the absence of long range order, of propagating phonon-like modes up to quite large scattering vectors. Specifically, in the large wavelength limit, both glasses and crystals behave as an elastic continuum and a well-defined frequency-wavevector relation exists for both longitudinal and transverse waves. However in glasses, owing to the lack of periodicity, the vibrational normal modes are not described by plane waves and the discrete translational symmetry cannot be invoked to assign well determined frequencies to a given wavevector and to define the Brillouin zone. Nevertheless glasses, although lacking long-range periodicity, possess some medium range order extending beyond the first neighbors and indeed longitudinal dispersion curves and a pseudo-Brillouin zone (p-BZ) have been experimentally identified in a variety of systems. ${ }^{1,10,19,22,41}$ The frequencies of these modes can be as high as a few terahertz and can exceed that of the "boson peak" (BP) found in glasses and related to an excess of vibrational modes over the Debye density of states. ${ }^{2,4}$ Similar results are found not only in the vitreous solid phase but also in the liquid

a)Electronic mail: giacomo.baldi@unitn.it one, demonstrating the persistence of a solid-like behavior at these high frequencies. ${ }^{11,38,41}$

For sufficiently large exchanged wavevectors, typically above a few $\mathrm{nm}^{-1}$, the situation becomes more complicated. $\mathrm{X}$-ray and neutron scattering experiments in some glasses ${ }^{10,12}$ and liquids ${ }^{42,43}$ have revealed the presence of a second weakly dispersive excitation. This second excitation has been assigned to a reminiscence of the transverse dynamics found in crystals on the basis of results from MD simulations, ${ }^{44}$ experiments performed on glasses, such as $\mathrm{SiO}_{2},{ }^{10,22}$ liquids, such as water, ${ }^{42,44,45}$ and on the corresponding crystals (quartz and ice). In any case, there is no general agreement on the nature of these excitations as well as on the existence of large wavevector transverse-like excitations in glasses and liquids; this is mainly because both neutrons and X-ray scattering techniques probe only longitudinal atomic displacements. The observation of a scattering contribution at the frequency of transverse modes is however possible at sufficiently large exchanged wavevectors if the mode polarization is not purely transverse, giving rise to what is often referred to as the mixing phenomenon. ${ }^{44,46}$

The aim of the present work is to achieve a better understanding of the collective excitations in 3-methylpentane (in the following 3MP) glass by studying in some detail the wavevector, $q$, dependence of their width. We have probed the vibrational excitations at terahertz frequencies by means of inelastic X-ray scattering. In the present contribution, we complement the results discussed in a recent paper ${ }^{41}$ by reporting new data measured below the glass temperature, $T_{g}$. We find that a more consistent description of the spectra can be achieved if we take into account the simultaneous presence of two vibrational modes. Since a similar behavior has 
TABLE I. Physical properties of a 3-methylpentane glass close to $T_{g}: v_{L}$, longitudinal sound velocity; $v_{T}$, transverse sound velocity measured using visible Brillouin light scattering, ${ }^{49} T_{g}$, glass transition temperature $;{ }^{50,51} T_{m}$, melting point temperature; $\rho$, mass density extrapolated below $97 \mathrm{~K} ;{ }^{52} n$, refractive index as obtained from the Clausius-Mossotti relation; $;{ }^{49}$ FSDP, the position of the first diffraction peak in the static structure factor.

\begin{tabular}{lcccccc}
\hline \hline$v_{L}(\mathrm{~m} / \mathrm{s})$ & $v_{T}(\mathrm{~m} / \mathrm{s})$ & $T_{g}(\mathrm{~K})$ & $T_{m}(\mathrm{~K})$ & $\rho\left(\mathrm{g} / \mathrm{cm}^{3}\right)$ & $\mathrm{n}$ & $\operatorname{FSDP}\left(\AA^{-1}\right)$ \\
\hline 2868 & 1354 & 77 & 110.3 & 0.8515 & 1.51 & 1.4 \\
\hline \hline
\end{tabular}

already been found in other glasses, namely, tetrahedral glasses such as silica $\left(\mathrm{SiO}_{2}\right)^{22}$ and silicon diselenide $\left(\mathrm{SiSe}_{2}\right),{ }^{23}$ we are led to believe that the existence of more than one excitation in the inelastic scattering spectra is a rather general characteristic common to very different types of glasses. Making use also of Raman scattering measurements, we give a consistent picture of the whole pattern of excitations in the first pseudo-Brillouin zone in glassy 3MP. Moreover, with the aid of a simple model, we relate quantitatively the width of the individual vibrational excitations to the width of the first sharp diffraction peak (FSDP) in the static structure factor.

\section{EXPERIMENTAL DETAILS}

The sample used in both inelastic $\mathrm{X}$ ray scattering (IXS) and Raman studies is 3MP $>99 \%$ purity supplied by Sigma Aldrich. 3MP is a structural isomer of hexane, molecular formula $\mathrm{C}_{6} \mathrm{H}_{14}$, formed by a pentane chain with a methyl group bonded to the third carbon atom. It belongs to a group of molecules referred to as branched-chain alkanes ${ }^{47}$ or as methylated alkanes ${ }^{48}$ it forms a non-polymeric glass obtained by cooling the liquid below the glass transition temperature. The physical properties of glassy 3-MP relevant for this work are reported in Table I.

The IXS experiment was performed at the high-energy resolution beam line ID16 of the European Synchrotron Radiation Facility in Grenoble, France. ${ }^{53}$ The X-ray beam has an incident energy of $E_{0}=21747 \mathrm{eV}$, corresponding to an overall energy resolution of about $1.5 \mathrm{meV}$. Further details on the experimental setup can be found in Ref. 41. The Raman scattering spectra, in both vertical (VV) and in horizontal-vertical (HV) polarization configurations, were obtained using a JobinYvon U1000 double monochromator. The wavelength of the incident light (argon laser) was $5145 \AA$. The resolution was $\sim 2 \mathrm{~cm}^{-1}(\sim 0.25 \mathrm{meV})$.

\section{DATA ANALYSIS}

\section{A. IXS analysis}

All the spectra show evident side peaks which are well defined below $q \sim 10 \mathrm{~nm}^{-1}$. The spectra also exhibit a quite intense central line with a line width comparable to that of the resolution profile. Figure 1 shows, as an example, the IXS experimental data of 3-MP for $T=63 \mathrm{~K}$ in the glassy phase at different $q$ values together with the best fitting functions. The spectra measured with different analyzers of the IXS spectrometer are put on a common intensity scale using as a reference the elastic scattering from a plexiglass sample.

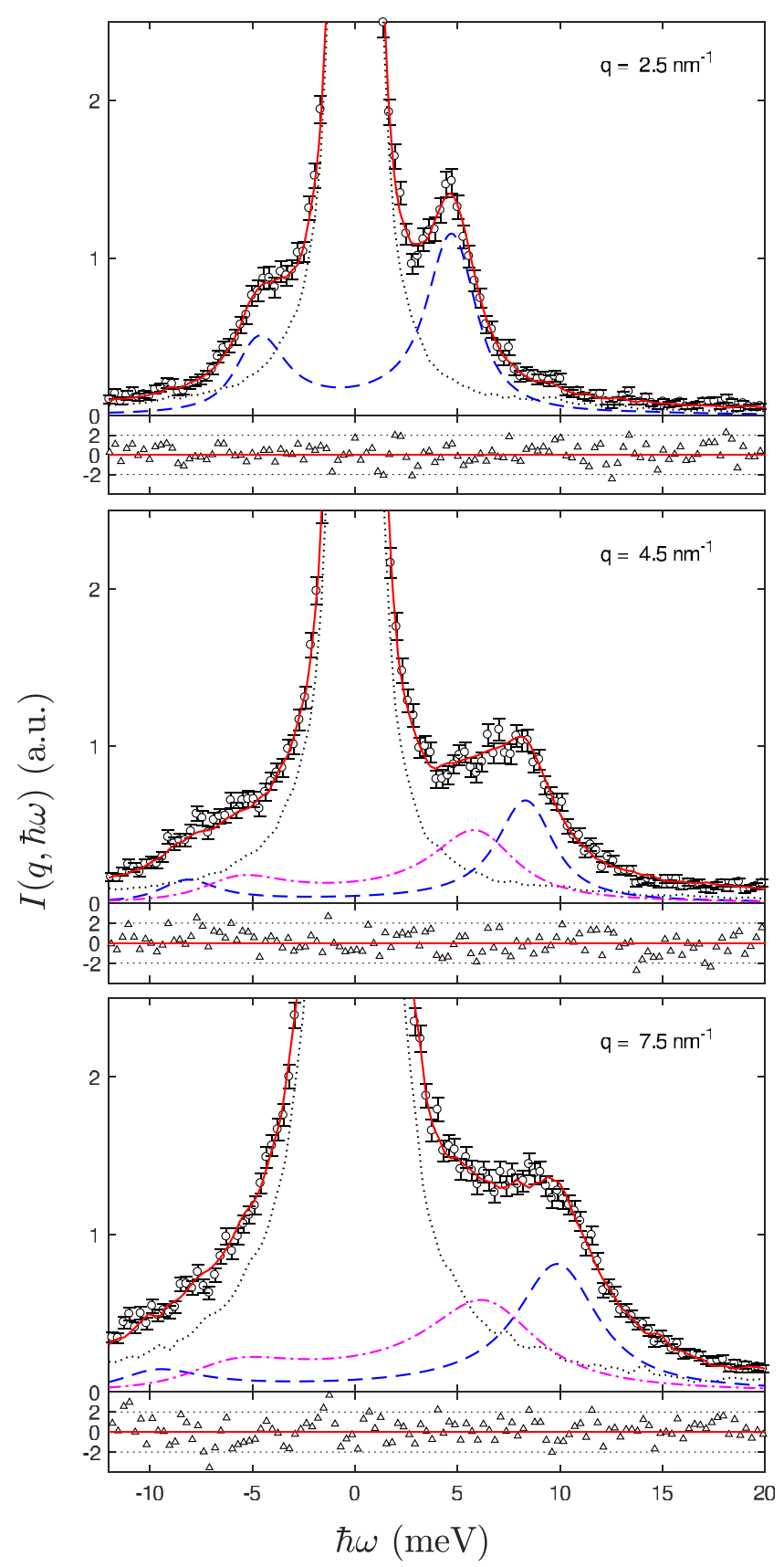

FIG. 1. IXS spectra of 3MP (black circles) measured in the glassy phase (T $=63 \mathrm{~K}$ ) for selected values of $q$. The upper panel is modeled with a single peak function for the inelastic part of the spectrum, while the bottom panels show two $q$ values where a double excitation model is more adequate. The plots include the instrumental transfer function (dotted black solid line), one (dashed blue) or two inelastic contributions to the fitting function (magenta dashed-dotted and blue dashed lines), while the reconstructed profile $I(q, \hbar \omega)$ is shown as a solid line (red). The baseline, $B$ in Eq. (1), is negligible in the scale of the figure. The residues are plotted in standard deviation units under the corresponding spectrum.

At low $q \mathrm{~s}\left(q<4 \mathrm{~nm}^{-1}\right)$, the spectra are well described by a single excitation model. Instead at higher $q \mathrm{~s}$, there is some evidence of additional inelastic intensity between the elastic line and the main inelastic peak, as shown in the two lower panels of the figure. Because of this, we have performed a new analysis of the IXS spectra using an elastic component superimposed to two damped harmonic oscillators (DHOs), extending the previous analysis in terms of a single DHO. ${ }^{41}$ 
Furthermore, we have added a new set of data that was not included in the previous analysis.

We account for the convolution with the measured resolution profile, $R(\omega)$, the detailed balance factor $f$,

$$
f(\omega, T)=\frac{\hbar \omega}{K T}\left[\frac{1}{1-\exp \left(-\frac{\hbar \omega}{K T}\right)}\right],
$$

and background, $B$, assuming

$$
\begin{aligned}
I(q, \omega)= & I_{\text {el }}(q, \omega)+I_{\text {inel }}(q, \omega) \propto R(\omega) \otimes[A \cdot \delta(\omega) \\
& +f(q, \omega) \cdot S(q, \omega)]+B .
\end{aligned}
$$

The elastic line, $I_{e l}(q, \omega)$, is proportional to the instrumental resolution function times $A$, a free parameter representing the intensity of the central line relative to that of the side peaks, while $S(q, \omega)$ is given by

$S(q, \omega)=\frac{1}{\pi} \operatorname{Re}\left\{\lim _{s \rightarrow i \omega}\left[\left(s+\frac{\omega_{L}^{2}}{s+\Gamma_{L}}\right)^{-1}+a\left(s+\frac{\omega_{T}^{2}}{s+\Gamma_{T}}\right)^{-1}\right]\right\}$.

The other physically relevant free fit parameters are as follows: the characteristic frequencies $\omega_{L}$ and $\omega_{T}$, the dampings $\Gamma_{L}$ and $\Gamma_{T}$, and the relative amplitude $a$ of the two modes. This expression reduces to the single DHO model if $a=0$. Some typical fits are shown in the lower panels of Fig. 1.

\section{B. Raman analysis}

The first order Raman scattering in the HV configuration, for a Stokes process, is connected to the vibrational density of states $g(\omega)^{54,55}$ by the equation

$$
I^{R}(\omega, T)=\frac{C(\omega) g(\omega)[n(\omega, T)+1]}{\omega} .
$$

Here $I^{R}(\omega, T)$ is the Raman intensity, $C(\omega)$ is the light to the vibration coupling function, $g(\omega)$ is the vibrational density of states, and $n(\omega, T)$ is the Bose-Einstein population factor. We introduce the reduced Raman intensity, $I_{\text {red }}^{R}$, to get rid of the trivial temperature dependence of $n(\omega, T)$,

$$
I_{\text {red }}^{R}(\omega, T)=\frac{I^{R}}{[n(\omega, T)+1] \omega}=\frac{C(\omega) g(\omega)}{\omega^{2}} .
$$

This quantity is plotted in Fig. 2(a), which shows the BP centered at about $15 \mathrm{~cm}^{-1}(\sim 1.9 \mathrm{meV})$. The frequency position of the peak was obtained by a local Gaussian fit given by the red line in the figure. Different theoretical models have been proposed for the frequency dependence of the coupling function $C(\omega)$ and, as shown in Ref. 55, the formal connection between the Raman intensity and the density of states is far from being simple. In the following, we will use the empirical relation $C(\omega) \sim \omega$ in the frequency region of the BP. It is however worth noting that in many systems, $C(\omega)$ tends to a constant value below the BP maximum. ${ }^{56,57}$ The linear frequency behavior has been observed in a variety of systems, both strong and fragile glasses, in network forming systems, and in polymeric ones, by comparing neutron and Raman scattering data. ${ }^{56,58,59}$ With this assumption, we obtain the following expression for the density of states:

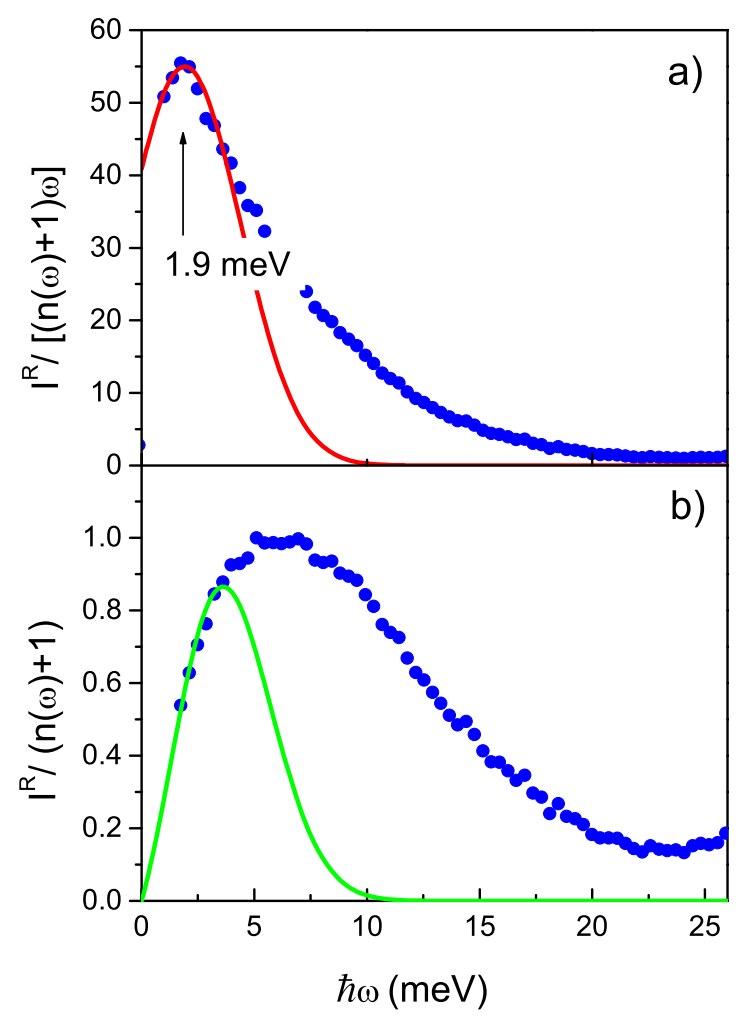

FIG. 2. Low frequency reduced Raman spectrum in the HV configuration (a) and density of states (b) of a 3-methylpentane glass. The line in panel (a) is a Gaussian fit around the maximum, to estimate the position of the BP; the green line in panel (b) is the same Gaussian fit multiplied by $\omega$.

$$
g(\omega) \sim \frac{I^{R}}{n(\omega, T)+1}=\omega I_{\text {red }}^{R} .
$$

The density of states determined using this expression for the Raman data of 3MP glass is shown in Fig. 2(b). The green line in Fig. 2(b) is the Gaussian fit of Fig. 2(a) multiplied for the frequency in order to show at which frequency the BP shifts when we report the density of states instead of the reduced density of states.

\section{RESULTS AND DISCUSSION}

\section{A. Single excitation model}

In the upper panel of Fig. 3, we report the width, $\Gamma$, of the inelastic features appearing in the IXS spectra of the glass phase $\left(T_{1}=63 \mathrm{~K}\right)$ determined by a single DHO analysis. In the same figure, we also report the values obtained just above the glass transition $\left(T_{2}=80 \mathrm{~K}\right)$ and in the liquid phase $\left(T_{3}\right.$ $=150 \mathrm{~K})$. The width increases markedly with temperature, indicating the presence of anharmonic effects. ${ }^{61,62}$ The lower panel of the figure shows the parameters $\Omega$ and $\Gamma$ at the lower temperature, $T_{1}=63 \mathrm{~K}$, corresponding to the glassy phase. In the low $q$ range, $q \lesssim 4 \mathrm{~nm}^{-1}$, the dispersion curve is approximately linear and the damping follows a quadratic $q$ dependence, $\Gamma \sim q^{2} .{ }^{63-67}$ For $q>4 \mathrm{~nm}^{-1}$, the dispersion curve goes through a maximum followed by a weak dispersion at even higher $q$ s. In the same $q$ range, the width of the inelastic peak deviates from the $\sim q^{2}$ law and follows the dispersion curve. The inset of the lower panel of Fig. 3 highlights 


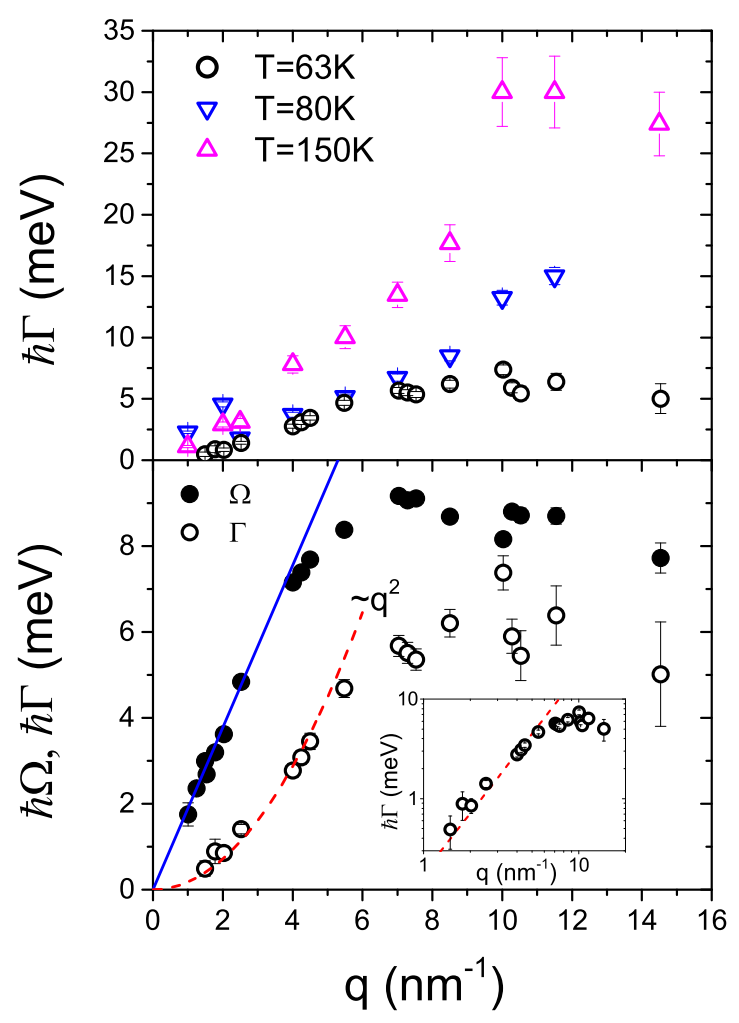

FIG. 3. Upper panel: width of the excitation versus $q$ at the three different temperatures indicated in the legend. Lower panel: $q$ dependence of the position and width of the single DHO model in the glassy phase, $T=63 \mathrm{~K}$. The inset shows the damping $\Gamma=\Gamma(q)$ in a log-log scale.

the $\Gamma \sim q^{2}$ law at low $q$ s in a log-log scale plot. The observed $q$ dependence of the position and width of the inelastic peak is quite common for a dielectric glass.
It is well documented that anharmonic effects play a major role in determining the damping of sound waves at the frequencies explored by ultrasound techniques and Brillouin light scattering spectroscopy. This happens typically at frequencies below $100 \mathrm{GHz}$ where the anharmonic contributions follow a quadratic wavevector dependence. However, as the exchanged wavevector is increased, larger frequencies are probed and relaxation processes can dramatically reduce the anharmonic contributions. In the terahertz regime, these tend to become negligible in comparison with the scattering due to the structural disorder. The frequency range where this elastic, temperature independent scattering becomes dominant may depend on the investigated sample. Indeed we have recently observed anharmonic temperature dependent effects on the width of the IXS inelastic peak in a sodium silicate glass at temperatures below the glass transition. ${ }^{60}$ It is therefore not surprising that, in the present case where the temperature is close to the glass transition point, we can find a $\Gamma \sim q^{2}$ behavior arising from a residual anharmonicity in the glassy phase.

\section{B. Double excitation model}

As mentioned previously, a second inelastic feature appears in the spectra when $q$ exceeds $4 \mathrm{~nm}^{-1}$. We have thus performed an analysis in terms of a two-excitation model as described in Sec. III. The evidence of the presence of two excitations is better appreciated by looking at the inelastic part of the spectra, as shown in Fig. 4. The figure shows the inelastic intensity at four $q$ values obtained by subtracting the elastic line, determined from the fitting procedure, from the measured intensity. The error bars take into account the uncertainties in

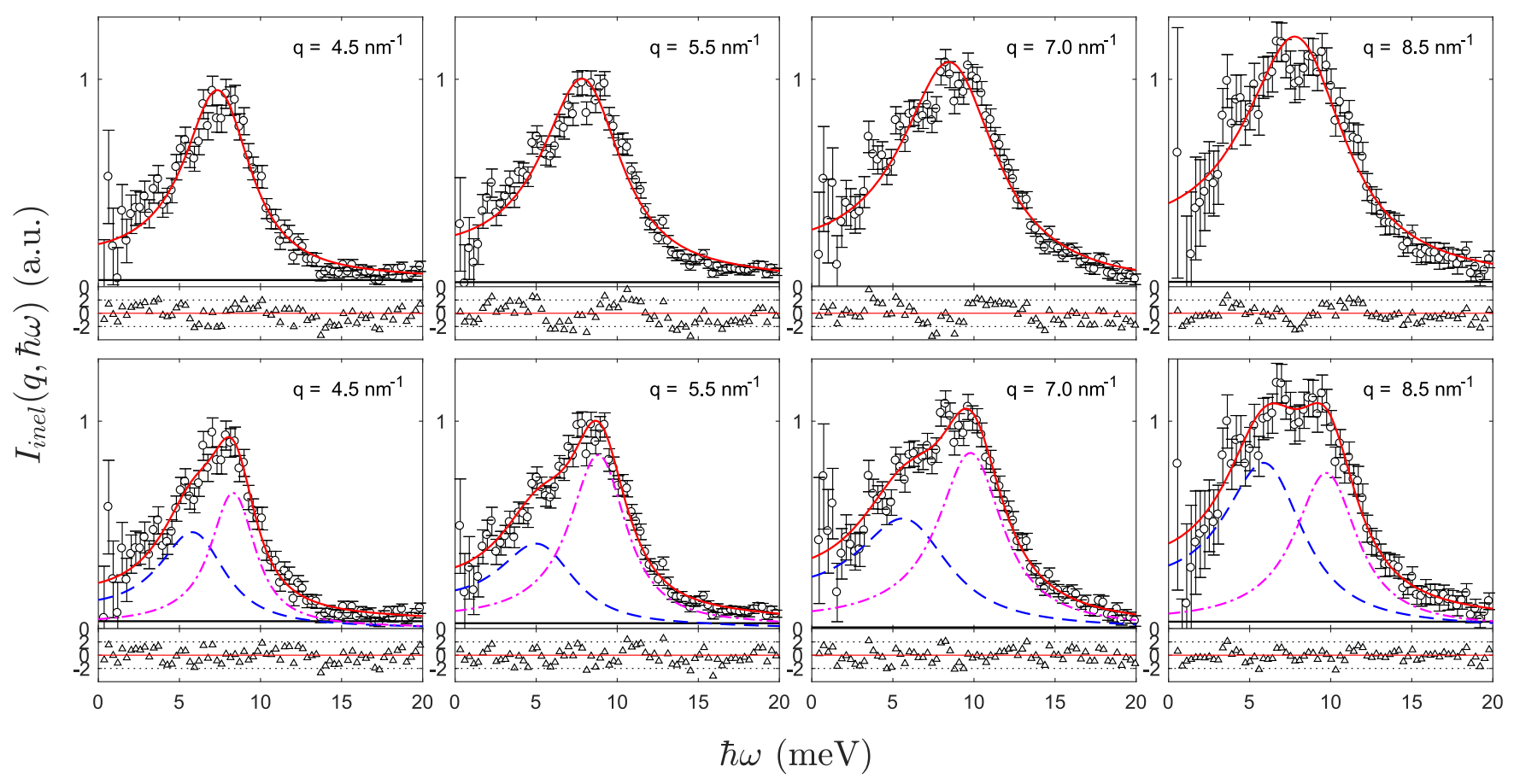

FIG. 4. Inelastic intensity of the IXS spectra of 3MP (black circles) measured in the glassy phase (T $=63 \mathrm{~K}$ ) for selected values of $q$. The upper panel shows the data points and the fit with a single DHO model. The lower panel shows the corresponding data and the fits with a double excitation model. The continuous line (red) is the fit, the dashed curve (blue) is the lower frequency peak, while the dashed-dotted (magenta) curve is the higher frequency one. The horizontal line (black) is the baseline, $B$ in Eq. (1). The residues are plotted in standard deviation units below each figure. 
the measured spectra, measured resolution profile, and determination of the elastic intensity. In the upper line of the figure, the peak is modeled as a single DHO, while the lower line shows the corresponding fit with two excitations. The residues between the model and the data are plotted below each spectrum in standard deviation units and allow appreciating the improvement in the fit when two excitations are used instead of one. This is particularly evident for the two central spectra in the figure, where the second excitation appears as a discernible side shoulder to the main inelastic peak. For $q$ values above $10 \mathrm{~nm}^{-1}$, the presence of a second (transverse) mode is hard to detect because the contrast between the inelastic and the elastic lines decreases as $q$ increases towards the peak of the static structure factor $S(q)$.

The values of $\hbar \omega_{L}$ and $\hbar \omega_{T}$ obtained from this fitting procedure are reported in Fig. 5 as a function of $q$ (left panel) together with the values of the single DHO frequency. We have parameterized the dispersion curves for both modes using a simple sinusoidal dispersion law of the form ${ }^{68}$

$$
E=\hbar \omega=\hbar v \frac{2 q_{0}}{\pi}\left|\sin \left(\frac{\pi q}{2 q_{0}}\right)\right| .
$$

This law is characterized by an initial slope, which depends on the apparent sound velocity $v$, being $E \sim \hbar v q$ in the limit of $q \rightarrow 0$, and by the occurrence of a maximum for $q$ $=q_{0}$. The $q_{0}$ values can be related to the pseudo-Brillouin zone (p-BZ) boundary wavevector characterized by a "lattice parameter" $a_{0}=\pi / q_{0}$, as discussed in Ref. 41 . We have determined the apparent sound velocities of the dispersion curves of the two excitations by performing a $\chi$ square minimization of Eq. (6), fixing the parameter $q_{0}$ to the value $(7.6 \pm 0.2) \mathrm{nm}^{-1}$ determined in Ref. 41 for the points of the single excitation model. The two sound velocities are $v_{L}=(3190 \pm 60) \mathrm{m} / \mathrm{s}$ and $v_{T}=(2060 \pm 80) \mathrm{m} / \mathrm{s}$. The higher frequency mode is presumably of predominant longitudinal character. On the contrary,

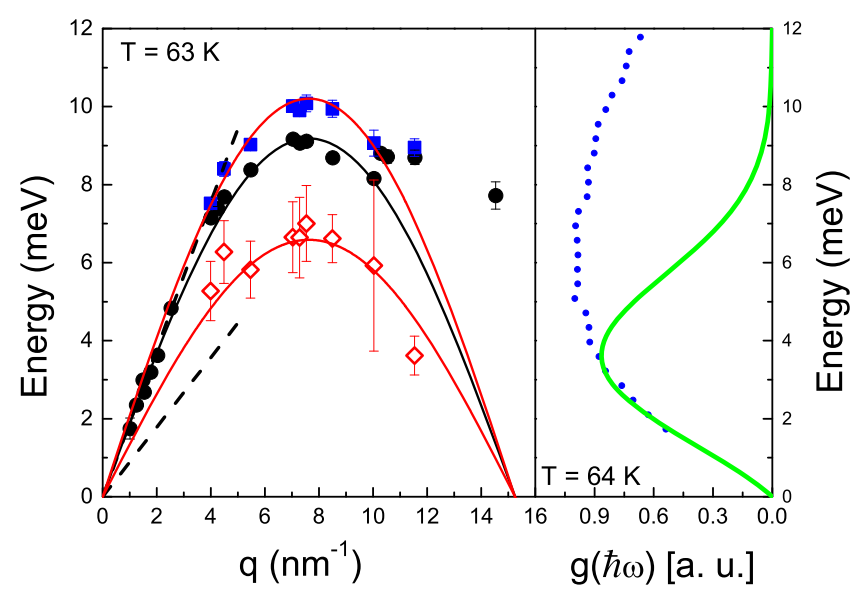

FIG. 5. (Left panel) Dispersion curve. Circles (black): single DHO fitting parameter $\hbar \omega_{0}$. The squares (blue) and the open diamonds (red) are, respectively, the parameters $\hbar \omega_{L}$ and $\hbar \omega_{T}$ of the double DHO model of Eq. (2). The continuous lines are the best fit of Eq. (6) to the three different data sets, as described in the text. The dashed lines correspond to the linear dispersion at the macroscopic scale, expected from the longitudinal and transverse sound velocities reported in Table I. (Right panel) Same data as in Fig. 2(b) in an expanded energy region. the lower frequency excitation shows a sound velocity significantly different from the macroscopic value of the transverse sound velocity. This discrepancy can be justified by the fact that what we measure is related to the longitudinal component of the modes belonging to the transverse branches. We will come back to this point in the following. We will use the terms "longitudinal" and "transverse" to label the two excitations just for convenience since they do not possess a welldefined polarization. In fact, the reason why we can observe the lower frequency mode in the IXS spectra is that its polarization presents a non-negligible projection on the longitudinal direction.

The energies of the longitudinal excitation determined by the two-peak model are only slightly higher than those of the single DHO model which are shown in Fig. 5 as circles. This can also be seen directly from the spectra in Fig. 4 where the single excitation model tends to underestimate the position of the higher frequency peak. It is worth noting that the sinusoidal dispersion does not work well for the $q$ points above approximately $10 \mathrm{~nm}^{-1}$ because the dispersion curve in the glass does not seem to decrease towards the center of an hypothetical second p-BZ. This behavior is different in the supercooled liquid and in the liquid phases, where the curve appears to be more of a sinusoidal shape, as detailed in Ref. 41.

Let us now consider the relation between the BP and the dispersion curves. In the last years, a very large number of theoretical $^{30,32,37,46,69,70}$ and experimental ${ }^{2,4,24,56,71,72}$ studies have been carried out to understand the nature of these modes. Several different models have been proposed. Without going over all of the proposed explanations, we cite the work of Taraskin and coauthors, ${ }^{32}$ who proposed a relation between the BP and the lowest energy van Hove singularity of the corresponding crystal. The striking similarity of the dynamics in glasses and their crystalline counterparts ${ }^{22}$ in terms of dispersion curves and of the BP has been demonstrated by some studies in the last years. ${ }^{8,9,23,45,73}$ The results of the present work, shown in the right panel of Fig. 5, is consistent with this explanation. In fact, the energy of the maximum of the density of states is located close to the acoustic excitations at the zone boundary. In particular, the position of the BP in the density of states (the green line) is close to the lower energy excitation. This evidence strongly supports, despite the extreme simplicity of the model used to extract the BP position, that the $\mathrm{BP}$ is originated primarily from transverse excitations.

Interestingly, the relative intensity of the transverse excitation with respect to the longitudinal one grows as $q$ is increased, as shown in Fig. 6. This observation is consistent with the idea that the excitations are more strongly affected by the local structural order of the glass as the wavelength is reduced towards the interatomic spacing. As $q$ is increased, the lower frequency excitation loses progressively its transverse character with a corresponding increase of its spectral weight.

\section{Broadening due to disorder}

The fact that the dispersion curve of the 3MP glass is reminiscent of that typical of a crystal ${ }^{41}$ and other evidences 


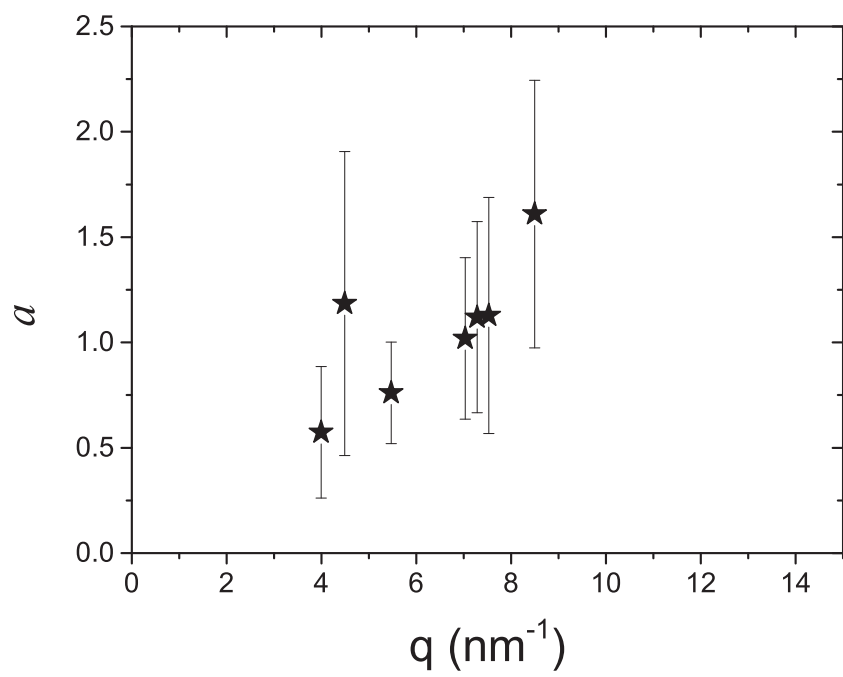

FIG. 6. Parameter $a$ of Eq. (2) as a function of $q$. $a$ is the ratio between the intensity of the transverse excitation to the longitudinal excitation.

in different systems ${ }^{22}$ suggest that the vibrational dynamics in the probed $q$ range is similar to that of a polycrystal. The spectrum of a polycrystal presents a continuum of modes corresponding to the frequencies of the vibrational branches of the corresponding single crystal in the various directions in reciprocal space. Moreover, since the polarization of the vibrational branches is purely longitudinal or purely transverse only in a few principal directions, all the acoustic and optic branches of a polycrystal may present a non-negligible projection along the longitudinal direction and thus give a finite contribution to the measured spectrum. Since the spectrum of a polycrystal is the result of an average of the single crystal spectrum along all possible directions in reciprocal space, this gives rise to a broadening of every vibrational branch.

The broadening mechanism present in a polycrystal and related to the anisotropy of the single crystal seems to occur also in glasses because of the residual order on the short and medium ranges. However, in a glass, the vibrational excitations are also broadened by a different mechanism, related to the structural disorder of the system. In this respect, we can imagine the vibrational dynamics of a glass to be that of a polycrystal in which the grain size is reduced to be of the order of a few interatomic distances. In order to account for the effect of disorder we can think of the first sharp diffraction peak, centered at $q_{\max }$, as reflecting the distribution of the Bragg peaks of a polycrystal. ${ }^{74}$ The idea is sketched in Fig. 7 where we plotted a Gaussian function to represent the FSDP of the glass and three dispersion curves obtained by varying the minimum of the curve within the full width at half maximum of the Gaussian function, $\Delta q_{\max }$. Hence we can model this broadening mechanism by considering a sinusoidal dispersion, Eq. (6), with a fixed sound velocity $v$ and varying the parameter $q_{0}$ between $q_{\max }^{-} / 2$ and $q_{\max }^{+} / 2$, where

$$
q_{\max }^{ \pm}=q_{\max }\left(1 \pm \frac{\Delta q_{\max }}{2 q_{\max }}\right) .
$$

This gives rise to a broadening of the vibrational branches because the dispersion curve can take all the possible

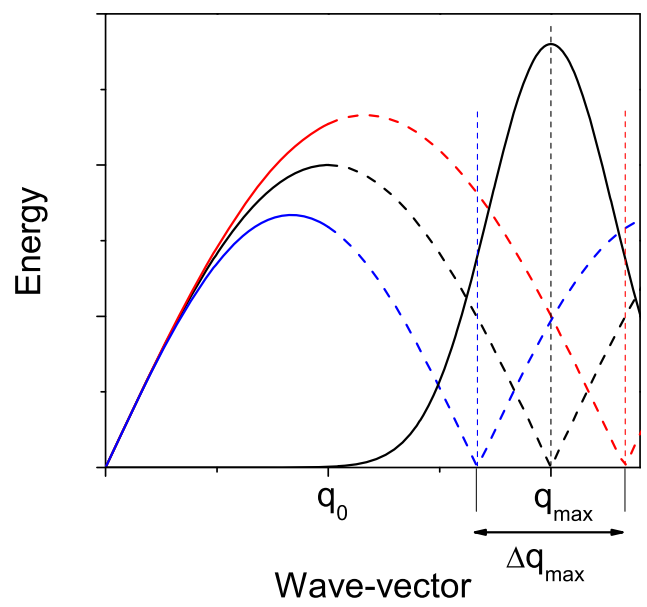

FIG. 7. Sketch of the mechanism that induces a broadening of the vibrational excitations of the glass because of the width of the FSDP. The sinusoidal curves are the dispersion at a fixed sound velocity but with different values of the zone boundary $q_{0}$. From top to bottom: $q_{0}=q_{\max }^{+} / 2, q_{0}=q_{\max } / 2$, and $q_{0}=q_{\max }^{-} / 2$, where $q_{\max }^{ \pm}$are defined in the text. The Gaussian curve centered at $q_{\max }$ describes the FSDP, and the vertical dashed lines are placed at the position of $q_{\max }$ and $q_{\max }^{ \pm}$.

values between the one with $q_{0}=q_{\max }^{-} / 2$ and the one with $q_{0}=q_{\max }^{+} / 2$. These two extremes are plotted in the sketch of Fig. 7 as the lower sinusoidal curve (blue) and the upper one (red), respectively. The resulting broadening is given by the following expressions, which contain the three parameters $v$, $q_{\max }$, and $\Delta q_{\max } / q_{\max }$ :

$$
\Delta \omega=\frac{v}{\pi}\left[q_{\max }^{+} \sin \left(\frac{\pi q}{q_{\max }^{+}}\right)-q_{\max }^{-} \sin \left(\frac{\pi q}{q_{\max }^{-}}\right)\right],
$$

for $q \leq q_{\max }^{-}$and

$$
\Delta \omega=\frac{v}{\pi} q_{\max }^{+} \sin \left(\frac{\pi q}{q_{\max }^{+}}\right),
$$

for $q_{\max }^{-}<q \leq q_{\max }$. The curves in Fig. 7 are plotted as dashed lines above the $\mathrm{p}$-BZ since the experimental dispersion curve becomes almost flat after this point.

As discussed in a previous paper, ${ }^{41}$ the value of $q_{0}$ determined from the dispersion curve of the single excitation model presents a slight discrepancy with respect to the value $q_{\max } / 2$ obtained from the FSDP. The discrepancy is of the order of $10 \%$ and is presumably related to the different kind of average in the reciprocal space that gives rise to the two observables. To make an estimate, we have used Eqs. (8) and (9) with $q_{\max }$ replaced by $2 q_{0}$ and $v$ equal to the parameters $v_{L}$ and $v_{T}$ determined in the analysis of the dispersion curves, as previously discussed. We have estimated the value of $\Delta q_{\max } / q_{\max }$ from a Gaussian fit of the static structure factor ${ }^{41}$ to be 0.37 . From this simple model, one obtains the curves plotted in Fig. 8 for the longitudinal (upper panel) and the transverse (lower panel) modes.

The estimate for the longitudinal mode accounts quantitatively and without free parameters for the damping at $q$ values close to the border of the first p-BZ. The good agreement suggests that this broadening mechanism is, in the system under study, dominant with respect to the one deriving from the average along the directions in reciprocal space. The model seems 


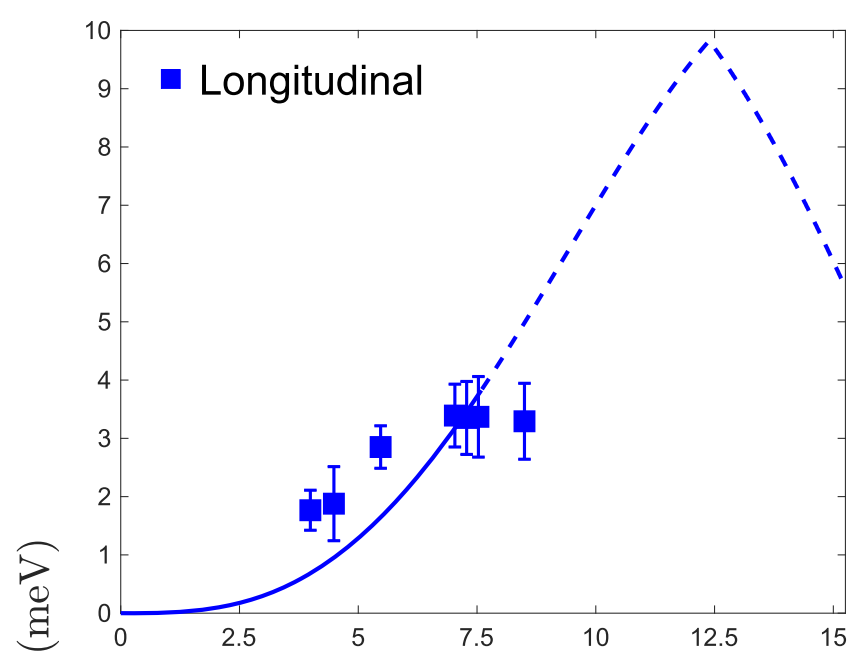

든

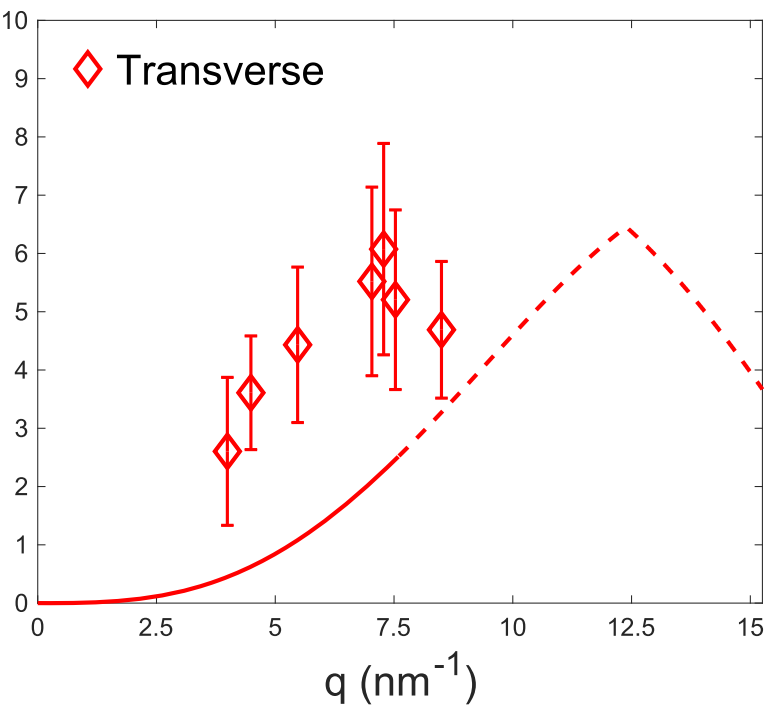

FIG. 8. Parameters $\hbar \Gamma_{L}$ (full blue squares, upper panel) and $\hbar \Gamma_{T}$ (open red diamonds, lower panel). The lines are computed from Eqs. (8) and (9) as detailed in the text. The lines are the estimate for the longitudinal mode (upper panel, blue) and for the transverse excitation (lower panel, red). The lines are plotted as dashed for $q>q_{0}$, see the text for details.

less accurate in describing the broadening of the transverse excitation. This could be due to the presence of two distinct transverse branches in a crystal and to the effect that this has on the apparent linewidths after averaging over different directions in the reciprocal space. The presence of two transverse branches could also explain the discrepancy between the initial slope of the lower dispersion curve in Fig. 5 and the transverse sound velocity reported in Table I. We thus expect the contribution to the damping coming from the local anisotropy of the glass to be more relevant for the transverse branch with respect to the longitudinal one. The curves in Fig. 8 are plotted as dashed lines for $q>q_{0}$ since above $q_{0}$ the model presumably overestimates the damping because the dispersion curve does not follow the sinusoidal law of Eq. (6). The model would predict a saturation of the damping to a constant value in the assumption that the dispersion curve flattens above the p-BZ.

It is worth noting that the model we propose, although phenomenological in nature, allows us to take into account the bending of the dispersion curves towards the border of the pseudo-Brillouin zone boundary. The theoretical models proposed up to now to describe the vibrational dynamics of glasses usually lack the ability to predict the width of the excitations in the high $q$ range close to the p-BZ boundary. This is the case, for instance, of models based on heterogeneous elasticity ${ }^{36}$ or on the jamming theory of glasses, ${ }^{39,40}$ which are able to predict many aspects of the vibrational dynamics of glasses, such as the presence of a boson peak and the existence of a Rayleigh scattering regime. Both assume that the elastic modulus is a function of frequency and not of the wavevector, an hypothesis which is not compatible with experimental data at high $q$ s. $^{75}$

\section{v. CONCLUSIONS}

Our experimental results, for $q$ larger than $4 \mathrm{~nm}^{-1}$, are consistent with the presence of at least two distinct modes in the IXS spectra of vitreous 3-MP. These are identified as arising from longitudinal and transverse excitations. Below $4 \mathrm{~nm}^{-1}$, the intensity of the transverse excitation is negligible with respect to the longitudinal one, as expected for an isotropic system in which the contribution of the longitudinal atomic displacements to the transverse excitations must vanish in the $q \rightarrow 0$ limit. The longitudinal excitation propagates with an apparent sound velocity determined by the initial slope of the dispersion curve, which turns out to be in good agreement with the longitudinal sound velocity. The dispersion reaches, for $q=q_{0}$, a maximum which can be related to the existence of a pseudo-Brillouin zone characterized by a "lattice parameter" $a_{0}=2 \pi / q_{0}$. The dispersion of the transverse excitation lies always below the longitudinal one and, even if it cannot be followed down to the linear region, it suggests an initial slope definitely lower than the longitudinal one but slightly larger than that expected for the transverse excitation. The vibrational density of states, as measured by low frequency Raman scattering, shows a maximum in correspondence with the flattening of the dispersion curves. In particular, the frequency at which the transverse excitations flatten suggests that transverse acoustic excitations are primarily responsible for the position of the BP.

The question regarding the origin of the width of the acoustic modes has also been addressed. We have introduced a simple model which quantifies the effect of disorder on the spectral linewidth of modes in terms of the width of the first diffraction peak. We have found that this model describes quantitatively the behavior of the longitudinal mode's width but underestimates that of the transverse one. Despite the crudeness of the model, this could be related to the fact that, at nanometric scale, vitreous systems preserve the anisotropy of the corresponding crystal. Since these effects are completely absent in our model, it could be that the presence of two distinct transverse branches gives rise to a more pronounced broadening of these excitations with respect to the longitudinal ones.

\section{ACKNOWLEDGMENTS}

We gratefully acknowledge the prolific debates and stimulating discussions with the late Professor M. Sampoli who 
has brought to our attention the interesting phenomenology of this glass-forming liquid.

${ }^{1}$ J. Leadbetter, J. Chem. Phys. 51, 779 (1969).

${ }^{2}$ U. Buchenau, N. Nücker, and A. J. Dianoux, Phys. Rev. Lett. 53, 2316 (1984).

${ }^{3}$ U. Buchenau, Yu. M. Galperin, V. L. Gurevich, D. A. Parshin, M. A. Ramos, and H. R. Schober, Phys. Rev. B 46, 2798 (1992).

${ }^{4}$ G. Carini, M. Federico, A. Fontana, and G. A. Saunders, Phys. Rev. B 47, 3005 (1993).

${ }^{5}$ P. Benassi, A. Fontana, W. Frizzera, M. Montagna, V. Mazzacurati, and G. Signorelli, Philos. Mag. B 71, 761 (1995).

${ }^{6}$ M. T. Dove, M. J. Harris, A. C. Hannon, J. M. Parker, I. P. Swainson, and M. Gambhir, Phys. Rev. Lett. 78, 1070 (1997).

${ }^{7}$ A. Matic, D. Engberg, C. Masciovecchio, and L. Börjesson, Phys. Rev. Lett. 86, 3803 (2001).

${ }^{8}$ M. Nakamura, M. Arai, Y. Inamura, T. Otomo, and S. M. Bennington, Phys. Rev. B 67, 064204 (2003).

${ }^{9}$ A. Matic, C. Masciovecchio, D. Engberg, G. Monaco, L. Börjesson, S. C. Santucci, and R. Verbeni, Phys. Rev. Lett. 93, 145502 (2004).

${ }^{10}$ B. Ruzicka, T. Scopigno, S. Caponi, A. Fontana, O. Pilla, P. Giura, G. Monaco, E. Pontecorvo, G. Ruocco, and F. Sette, Phys. Rev. B 69, 100201 (2004).

${ }^{11}$ T. Scopigno, G. Ruocco, and F. Sette, Rev. Mod. Phys. 77, 881 (2005).

${ }^{12}$ L. E. Bove, E. Fabiani, A. Fontana, F. Paoletti, C. Petrillo, O. Pilla, and I. C. V. Bento, Europhys. Lett. 71, 563 (2005).

${ }^{13}$ M. D. Ruiz-Martín, M. Jiménez-Ruiz, F. J. Bermejo, and R. Fernández-Perea, Phys. Rev. B 73, 094201 (2006).

${ }^{14}$ S. Caponi, A. Fontana, F. Rossi, G. Baldi, and E. Fabiani, Phys. Rev. B 76, 092201 (2007)

${ }^{15}$ G. Baldi, V. M. Giordano, G. Monaco, F. Sette, E. Fabiani, A. Fontana, and G. Ruocco, Phys. Rev. B 77, 214309 (2008).

${ }^{16}$ E. Fabiani, A. Fontana, and U. Buchenau, J. Chem. Phys. 128, 244507 (2008).

${ }^{17}$ G. Baldi, A. Fontana, G. Monaco, L. Orsingher, S. Rols, F. Rossi, and B. Ruta, Phys. Rev. Lett. 102, 195502 (2009).

${ }^{18}$ M. Zanatta, G. Baldi, S. Caponi, A. Fontana, E. Gilioli, M. Krish, C. Masciovecchio, G. Monaco, L. Orsingher, F. Rossi, G. Ruocco, and R. Verbeni, Phys. Rev. B 81, 212201 (2010).

${ }^{19}$ L. Orsingher, G. Baldi, A. Fontana, L. E. Bove, T. Unruh, A. Orecchini, C. Petrillo, N. Violini, and F. Sacchetti, Phys. Rev. B 82, 115201 (2010).

${ }^{20}$ B. Rossi, S. Caponi, F. Castiglione, S. Corezzi, A. Fontana, M. Giarola, G. Mariotto, A. Mele, C. Petrillo, F. Trotta, and G. Viliani, J. Phys. Chem. B 116, 5323 (2012).

${ }^{21}$ B. Ruta, G. Baldi, F. Scarponi, D. Fioretto, V. M. Giordano, and G. Monaco, J. Chem. Phys. 137, 214502 (2012).

${ }^{22}$ G. Baldi, M. Zanatta, E. Gilioli, V. Milman, K. Refson, B. Wehinger, B. Winkler, A. Fontana, and G. Monaco, Phys. Rev. Lett. 110, 185503 (2013).

${ }^{23}$ M. Zanatta, A. Fontana, A. Orecchini, C. Petrillo, and F. Sacchetti, J. Phys. Chem. Lett. 4, 1143 (2013).

${ }^{24}$ A. I. Chumakov, G. Monaco, A. Fontana, A. Bosak, R. P. Hermann, D. Bessas, B. Wehinger, W. A. Crichton, M. Krisch, R. Rüffer, G. Baldi, G. Carini, Jr., G. Carini, G. D’Angelo, E. Gilioli, G. Tripodo, M. Zanatta, B. Winkler, V. Milman, K. Refson, M. T. Dove, N. Dubrovinskaia, L. Dubrovinsky, R. Keding, and Y. Z. Yue, Phys. Rev. Lett. 112, 025502 (2014).

${ }^{25}$ C. Weigel, M. Foret, B. Hehlen, M. Kint, S. Clement, A. Polian, R. Vacher, and B. Rufflé, Phys. Rev. B 93, 224303 (2016).

${ }^{26}$ G. Baldi, G. Carini, Jr., G. Carini, A. Chumakov, R. Dal Maschio, G. D'Angelo, A. Fontana, E. Gilioli, G. Monaco, L. Orsingher, B. Rossi, and M. Zanatta, Philos. Mag. 96, 754 (2016).

${ }^{27}$ V. G. Karpov, M. I. Klinger, and F. N. Ignat'ev, Sov. Phys. JETP 57, 439 (1983), available at http://www.jetp.ac.ru/cgi-bin/e/index/e/57/2/ p439?a=list.

${ }^{28}$ W. Jin, P. Vashishta, R. K. Kalia, and J. P. Rino, Phys. Rev. B 48, 9359 (1993).

${ }^{29}$ S. N. Taraskin and S. R. Elliott, Phys. Rev. B 56, 8605 (1997).

${ }^{30}$ W. Schirmacher, G. Diezemann, and C. Ganter, Phys. Rev. Lett. 81, 136 (1998).

31 J. L. Feldman, P. B. Allen, and S. R. Bickham, Phys. Rev. B 59, 3551 (1999).

${ }^{32}$ S. N. Taraskin, Y. L. Loh, G. Natarajan, and S. R. Elliott, Phys. Rev. Lett. 86, 1255 (2001).

${ }^{33}$ J. W. Kantelhardt, S. Russ, and A. Bunde, Phys. Rev. B 63, 064302 (2001).
${ }^{34}$ T. S. Grigera, V. Martin-Mayor, G. Parisi, and P. Verrocchio, J. Phys.: Condens. Matter 14, 2167 (2002).

${ }^{35}$ V. Gurarie and A. Altland, Phys. Rev. Lett. 94, 245502 (2005).

${ }^{36}$ W. Schirmacher, G. Ruocco, and T. Scopigno, Phys. Rev. Lett. 98, 025501 (2007).

${ }^{37}$ D. A. Parshin, H. R. Schober, and V. L. Gurevich Phys. Rev. B 76, 064206 (2007).

${ }^{38}$ V. V. Brazkin and K. Trachenko, J. Phys. Chem. B 118, 11417 (2014).

${ }^{39}$ E. DeGiuli, A. Laversanne-Finot, G. Düring, E. Lerner, and M. Wyart, Soft Matter 10, 5628 (2014).

${ }^{40}$ E. DeGiuli, E. Lerner, C. Brito, and M. Wyart, Proc. Natl. Acad. Sci. U. S. A. 111, 17054 (2014)

${ }^{41}$ P. Benassi, M. Nardone, A. Giugni, G. Baldi, and A. Fontana, Phys. Rev. B 92, 104203 (2015).

${ }^{42}$ G. Ruocco, F. Sette, U. Bergmann, M. Krisch, C. Masciovecchio, V. Mazzacurati, G. Signorelli, and R. Verbeni, Nature 379, 521 (1996).

${ }^{43}$ S. Hosokawa, M. Inui, Y. Kajihara, K. Matsuda, T. Ichitsubo, W.-C. Pilgrim, H. Sinn, L. E. González, D. J. González, S. Tsutsui, and A. Q. R. Baron, Phys. Rev. Lett. 102, 105502 (2009).

${ }^{44}$ M. Sampoli, G. Ruocco, and F. Sette, Phys. Rev. Lett. 79, 1678 (1997).

${ }^{45}$ E. Pontecorvo, M. Krisch, A. Cunsolo, G. Monaco, A. Mermet, R. Verbeni, F. Sette, and G. Ruocco, Phys. Rev. E 71, 011501 (2005).

${ }^{46}$ O. Pilla, L. Angelani, A. Fontana, J. R. Gonçalves, and G. Ruocco, J. Phys.: Condens. Matter 15, S995 (2003).

${ }^{47}$ S. Shahriari, A. Mandanici, L. M. Wang, and R. Richter, J. Chem. Phys. 121, 8960 (2004).

${ }^{48}$ K. L. Ngai, Phys. Rev. B 71, 214201 (2005).

${ }^{49}$ P. Benassi, M. Nardone, and A. Giugni, J. Chem. Phys. 137, 094504 (2012).

${ }^{50}$ A. C. Ling and J. E. Willard, J. Phys. Chem. 72, 1918 (1968).

${ }^{51}$ H. L. Finke and J. F. Messerly, J. Chem. Thermodyn. 5, 247 (1973).

${ }^{52}$ A. A. Ruth, B. Nickel, and H. Lesche, Z. Phys. Chem. 175, 91 (1992).

${ }^{53}$ C. Masciovecchio, U. Bergmann, M. H. Krisch, G. Ruocco, F. Sette, and R. Verbeni, Nucl. Instrum. Methods 111, 181 (1996).

${ }^{54}$ F. L. Galeener and P. N. Sen, Phys. Rev. B 17, 19228 (1978).

${ }^{55}$ B. Schmid and W. Schirmacher, Phys. Rev. Lett. 100, 137402 (2008).

${ }^{56}$ A. Fontana, R. Dell'Anna, M. Montagna, F. Rossi, G. Viliani, G. Ruocco, M. Sampoli, and U. Buchenau, Europhys. Lett. 47, 56 (1999).

${ }^{57}$ A. P. Sokolov, U. Buchenau, W. Steffen, B. Frick, and A. Wischnewski, Phys. Rev. B 52, R9815(R) (1995).

${ }^{58}$ N. V. Surovtsev, and A. P. Sokolov, Phys. Rev. B 66, 054205 (2002).

${ }^{59}$ A. Fontana, F. Rossi, G. Viliani, S. Caponi, E. Fabiani, G. Baldi, G. Ruocco, and R. Dal Maschio, J. Phys.: Condens. Matter 19, 205145 (2007).

${ }^{60}$ G. Baldi, V. M. Giordano, B. Ruta, R. Dal Maschio, A. Fontana, and G. Monaco, Phys. Rev. Lett. 112, 125502 (2014).

${ }^{61}$ T. Scopigno, J.-B. Suck, R. Angelini, F. Albergamo, and G. Ruocco, Phys. Rev. Lett. 96, 135501 (2006).

${ }^{62}$ E. Guarini, U. Bafile, F. Barocchi, A. De Francesco, E. Farhi, F. Formisano, A. Laloni, A. Orecchini, A. Polidori, M. Puglini, and F. Sacchetti, Phys. Rev. B 88, 104201 (2013).

${ }^{63}$ C. Masciovecchio, G. Baldi, S. Caponi, L. Comez, S. Di Fonzo, D. Fioretto, A. Fontana, A. Gessini, S. C. Santucci, F. Sette, G. Viliani, P. Vilmercati, and G. Ruocco, Phys. Rev. Lett. 97, 035501 (2006).

${ }^{64}$ P. Benassi, S. Caponi, R. Eramo, A. Fontana, A. Giugni, M. Nardone, M. Sampoli, and G. Viliani, Phys. Rev. B 71, 172201 (2005).

${ }^{65}$ W. Dietsche and H. Kinder, Phys. Rev. Lett. 43, 1413 (1979).

${ }^{66}$ V. I. Kozub, A. M. Rudin, and H. R. Schober, Phys. Rev. B 50, 6032 (1994).

${ }^{67}$ B. Rufflé, D. A. Parshin, E. Courtens, and R. Vacher, Phys. Rev. Lett. 100, 015501 (2008).

${ }^{68}$ C. Kittel, Introduction to Solid State Physics (John Wiley and Sons, New York, 1966).

${ }^{69}$ E. Duval, A. Boukenter, and T. Achibat, J. Phys.: Condens. Matter 2, 10227 (1990).

${ }^{70}$ T. Grigera, V. Martin-Mayor, G. Parisi, and P. Verrocchio, Nature 422, 289 (2003).

${ }^{71}$ M. Foret, R. Vacher, E. Courtens, and G. Monaco, Phys. Rev. B 66, 024204 (2002).

${ }^{72}$ K. Niss, B. Begen, B. Frick, J. Ollivier, A. Beraud, A. Sokolov, V. N. Novikov, and C. Alba-Simionesco, Phys. Rev. Lett. 99, 055502 (2007).

${ }^{73}$ H. Shintani and H. Tanaka, Nat. Mater. 7, 870 (2008).

${ }^{74}$ V. M. Giordano and G. Monaco, Proc. Natl. Acad. Sci. U. S. A. 107, 21985 (2010).

${ }^{75}$ G. Baldi, V. M. Giordano, B. Ruta, and G. Monaco, Phys. Rev. B 93, 144204 (2016). 